\title{
Itinerarios que relacionan educación con trabajo. Revisión de conceptos, investigación y debates políticos 1
}

\author{
David Raffe \\ The University of Edinburgh. Centre for Educational Sociology (CES) \\ david.raffe@ed.ac.uk
}

\section{Resumen}

Este artículo examina de forma crítica la investigación y los debates políticos transnacionales sobre los itinerarios que relacionan educación con trabajo, y utiliza este examen para reflexionar sobre la utilidad del propio concepto de itinerarios. Identifica tres utilizaciones principales del concepto: para analizar la dimensión relativa y el papel de la educación académica, la formación profesional reglada y el aprendizaje, en un sistema de transición efectivo; para examinar las relaciones y las interconexiones entre itinerarios, y para evaluar de qué manera los itinerarios pueden reflejar las perspectivas y las prioridades de cada individuo joven y permitirle gestionar y ejercer control sobre sus propios itinerarios. Los itinerarios pueden ser útiles como concepto estructurador que vincula la política a la investigación, siempre que la metáfora se utilice con precisión y se eviten sus significados no intencionales.

Palabras clave: transición escuela-trabajo; itinerarios formativos; política educativa.

Abstract. Pathways Linking Education and Work: A Review of Concepts, Research, and Policy Debates

This paper reviews cross-national research and policy debates about pathways between education and work, and draws on this review to reflect on the utility of the pathways concept itself. It identifies three main uses of the concept: to analyse the relative size and role of gender education, school-based vocational education and apprenticeship, respec-

1. Nota de edición: el presente artículo fue publicado en inglés con el título «Pathways Linking Education and Work: A Review of Concepts, Research, and Policy Debates», en la revista Journal of Youth Studies, 6 (1), marzo de 2003, 3-19. Por el interés que pueda tener de acuerdo con la temática de este monográfico, nos ha parecido oportuno publicarlo, con las autorizaciones convenientes de la revista y del autor. Además, hemos pedido al autor que haga una actualización de su análisis y de sus propuestas para nuestra revista, que es el segundo texto que se presenta. Dicho segundo texto es inèdito y está realizado con la finalidad de completar el primer artículo. 
tively, in an effective transition system; to examine the relationships and interconnections between pathways; and to consider how pathways can reflect the perspective and priorities of individual young people and enable them to manage and control their own itineraries. Pathways can be useful as an organizing concept linking policy and research, provided that the metaphor is used with precision and its unintended meanings are avoided.

Key words: school to work transition; educational pathways; educational policy.

\begin{tabular}{|c|c|}
\hline \multicolumn{2}{|c|}{ Sumario } \\
\hline Introducción: la metáfora & Itinerarios y navegación \\
\hline de los itinerarios & Conclusión: puntos fuertes y débiles \\
\hline Comparando las principales & del concepto de itinerarios \\
\hline clases de itinerarios & Agradecimientos \\
\hline $\begin{array}{r}\text { Relaciones e interconexiones } \\
\text { entre itinerarios }\end{array}$ & Referencias bibliográficas \\
\hline
\end{tabular}

\section{Introducción: la metáfora de los itinerarios}

Las metáforas del viaje y del movimiento han impregnado la política y la literatura de la investigación sobre las transiciones de los jóvenes. Los encargados de formular las políticas y los académicos proyectan caminos reales y vías alternativas, pistas rápidas y carreteras de circunvalación, puentes y aparcamientos, viajes en tren y en coche. Evans y Furlong (1997) utilizaron cuatro metáforas para definir las fases a través de las cuales se han desarrollado la investigación y la política. En la década de 1960, la metáfora consistía en rellenar los «huecos» ${ }^{2}$ de la sociedad; el énfasis se puso en cumplir con las "tareas de crecimiento» ${ }^{3}$, con el objeto de garantizar una buena integración en los papeles de adultos. A medida que el paro juvenil fue aumentando y la participación en la educación se fue ampliando, surgió el concepto de itinerarios para describir los procesos más largos y complejos de transición que se estaban desarrollando. En la década de 1980, el concepto de trayectoria fue empleado para expresar las fuerzas sociales que configuraban las transiciones con mayor fuerza que los itinerarios diseñados por el gobierno. Esto significaba que los jóvenes que efectuaban la transición se diferenciaban en cuanto a sus recursos y en su ímpetu. Y en la década de 1990, surgieron las metáforas de las "navegaciones» ${ }^{4}$, a fin de expresar el papel activo de los individuos en la configuración de sus vidas con las oportunidades y los imperativos a los que se enfrentaban.

De todas estas metáforas, la de los itinerarios ha sido especialmente influyente en los debates transnacionales de política y en los responsables nacionales

2. N. de la t.: en la versión original, niches.

3. N. de la t.: en la versión original, growth tasks.

4. N. de la t.: en la versión original, navigations. 
de formular las políticas de educación. La Organización para la Cooperación y el Desarrollo Económico (OCDE) utilizó el concepto de itinerarios como principio estructurador de su actividad de educación y formación profesional y técnica en el período 1991-1996, así como de su análisis temático de la transición de la educación inicial a la vida laboral en el período 1997-1999 (OCDE, 1998, 2000). Los debates de política nacional también han sido formulados en términos de itinerarios, en particular en los países anglófonos como Australia e Inglaterra (Dearing, 1996; Kirby, 2000; Eldridge, 2001), pero también en otros países como Holanda, Noruega, Alemania y Dinamarca, cuya reforma de la educación y de la formación profesional (EFP) en el año 2000 se inspiró en el concepto de itinerarios (Nielsen, 2000: 67-68). Por otra parte, los científicos sociales académicos han sido más críticos con el concepto. Al situar los itinerarios como la segunda de sus cuatro metáforas, Evans y Furlong entienden que ésta ha sido desplazada por los enfoques posteriores, pero la principal fuerza de su crítica no consiste en que el concepto de itinerarios esté anticuado, sino en que ignora los aspectos de las transiciones de los jóvenes que se expresan en las metáforas de las trayectorias y las navegaciones. El concepto de itinerarios ha suscitado tres críticas principales de los técnicos académicos (Evans y Furlong, 1997; Dwyer y Wyn, 1998; Cohen y Ainley, 2000):

- Linealidad: ignora la complejidad, implica una clara diferencia entre itinerarios educativos y metas del mercado laboral, no tiene en cuenta el solapamiento de estudio y trabajo y supone que todas las transiciones van en la misma dirección.

- Economicismo: se centra en las transiciones a través de la educación al mercado laboral y deja de lado otras transiciones como las de familia, de vivienda o de estilo de vida.

- Individualismo: ignora la estructura social y la desigualdad, implica que los itinerarios son igualmente accesibles para cada persona y que si las personas difieren sistemáticamente en la forma en que los utilizan, esto es exclusivamente porque quieren llegar a lugares distintos.

A pesar de estas críticas, el concepto de itinerarios puede ser útil como concepto estructurador en los debates actuales sobre les transiciones del mundo de la educación al del trabajo, así como un puente que vincula la política tanto a la investigación empírica como a los debates teóricos actuales en las ciencias sociales. En este artículo, espero poder demostrar la utilidad del concepto de itinerarios mediante su utilización para estructurar un análisis crítico de la investigación y los debates políticos sobre las transiciones de la educación al trabajo.

Mi punto de partida es que los itinerarios son una metáfora. No son ni una teoría ni una herramienta analítica rigurosa. Una metáfora es un medio para transmitir significados, y la metáfora de los itinerarios es rica en significados. Tal como Evans y Furlong sugieren, adquirió popularidad porque expresaba la longitud y la complejidad de los itinerarios modernos; un itinerario es más largo que un simple paso, y los itinerarios pueden interconectarse. Otra razón 
de su popularidad entre los encargados de formular políticas es que refleja una perspectiva política; los itinerarios pueden construirse deliberadamente para conducir a destinos específicos, y pueden ser diseñados de tal forma que ciertos destinos sean más fáciles de alcanzar que otros. Aun cuando los itinerarios se han desarrollado de forma no planificada, pueden ser reconstruidos o modificados. Al mismo tiempo, la metáfora refleja el discurso individualista de los debates políticos actuales, y esto podría explicar su popularidad, no sólo en los países anglófonos con una tradición educativa liberal, sino también en países como Dinamarca y Alemania, que intentan proporcionar «itinerarios de formación» más «individualizados» (Ertl, 2002: 54). Los viajeros pueden escoger entre itinerarios distintos, y pueden cambiar de dirección, o pueden ponerse en camino sin ningún destino claro en mente.

Las metáforas tienen sus limitaciones. Pueden transmitir significados no deliberados. Las tres críticas que ya hemos mencionado - economicismo, linealidad e individualismo - se refieren a suposiciones implícitas que la gente puede hacer si utiliza el concepto de itinerarios de forma irreflexiva. Por otra parte, la metáfora de los itinerarios es imprecisa. Se puede utilizar de diversas maneras y para expresar diversos significados. La solución no es renunciar al concepto, sino ser más precisos y conscientes de nosotros mismos cuando lo utilicemos. La metáfora de los itinerarios tiende a ser utilizada en la investigación transnacional y en los análisis políticos de tres formas principales:

1. Para distinguir las principales clases de itinerarios más allá de la escolaridad obligatoria y de integración en el mercado laboral, para comparar sus puntos fuertes y débiles y para determinar su magnitud relativa y su papel en un sistema de transición efectivo.

2. Para examinar las relaciones e interconexiones entre itinerarios y para determinar cuál sería la mejor manera de organizar los sistemas de itinerarios.

3. Para analizar las relaciones entre itinerarios y las «navegaciones» de la gente joven que los utiliza; en particular, para evaluar de qué forma se podrían organizar los itinerarios para que permitiesen expresar las perspectivas y las prioridades de cada individuo joven, así como gestionar y ejercer un control sobre sus propios itinerarios.

Seguidamente, en este artículo, argumento sobre estas tres utilizaciones del concepto de itinerarios, identifico las preguntas políticas que típicamente están vinculadas a cada una y examino de forma crítica los debates relevantes de investigación y de política educativa. Me centraré en los países de la OCDE y en los itinerarios existentes entre educación y mercado laboral. El cuadro 1 proporciona una perspectiva general sobre ello.

\section{Comparando las principales clases de itinerarios}

La primera utilización de la metáfora de los itinerarios es la descripción y la comparación de las principales opciones educativas después de la educación obligatoria. Un ejemplo reciente de ello es el análisis temático de 
Cuadro 1. Tres utilizaciones del concepto de itinerario para vincular la investigación a la política

\begin{tabular}{|c|c|c|c|}
\hline $\begin{array}{l}\text { Utilización } \\
\text { del concepto } \\
\text { de itinerario }\end{array}$ & $\begin{array}{l}\text { Para comparar } \\
\text { las principales clases } \\
\text { de itinerarios }\end{array}$ & $\begin{array}{l}\text { Para examinar las } \\
\text { relaciones entre } \\
\text { itinerarios }\end{array}$ & $\begin{array}{l}\text { Para analizar } \\
\text { de qué forma los } \\
\text { itinerarios influyen } \\
\text { sobre la utilización } \\
\text { activa de éstos por } \\
\text { parte de los jóvenes. }\end{array}$ \\
\hline $\begin{array}{l}\text { Preguntas usuales } \\
\text { de política educativa }\end{array}$ & $\begin{array}{l}\text { ¿Cuál es la mejor } \\
\text { mezcla de itinerarios: } \\
\text { académico, } \\
\text { profesional } \\
\text { o no reglado? } \\
\text { ¿Cuál es la que } \\
\text { mejor secunda las } \\
\text { transiciones al mundo } \\
\text { laboral? }\end{array}$ & $\begin{array}{l}\text { ¿Deberían los } \\
\text { itinerarios ser } \\
\text { distintos y separados, } \\
\text { o relacionados o } \\
\text { unificados? ¿De qué } \\
\text { forma los itinerarios } \\
\text { se conectan como } \\
\text { sistema para favorecer } \\
\text { los modelos de } \\
\text { participación } \\
\text { deseados? }\end{array}$ & $\begin{array}{l}\text { ¿De qué forma los } \\
\text { itinerarios pueden } \\
\text { reflejar las prioridades } \\
\text { y las perspectivas de } \\
\text { los jóvenes? ¿De qué } \\
\text { forma los itinerarios } \\
\text { pueden estimular la } \\
\text { acción individual? }\end{array}$ \\
\hline $\begin{array}{l}\text { Problemas } \\
\text { contextuales }\end{array}$ & $\begin{array}{l}\text { Presiones hacia la } \\
\text { deriva académica } \\
\text { Contextos nacionales } \\
\text { que varían (por } \\
\text { ejemplo: factores } \\
\text { determinantes de } \\
\text { la demanda de } \\
\text { competencias del } \\
\text { mercado laboral) }\end{array}$ & $\begin{array}{l}\text { Presiones para la } \\
\text { unificación: presiones } \\
\text { económicas y sociales } \\
\text { cambiantes; expansión } \\
\text { y mayor complejidad } \\
\text { de los sistemas } \\
\text { educativos }\end{array}$ & $\begin{array}{l}\text { Individualización } \\
\text { Preocupación por } \\
\text { la ciudadanía activa. }\end{array}$ \\
\hline $\begin{array}{l}\text { Mensajes típicos } \\
\text { de la investigación }\end{array}$ & $\begin{array}{l}\text { El «mejor» itinerario } \\
\text { depende de las } \\
\text { circunstancias } \\
\text { nacionales / } \\
\text { Es deseable una } \\
\text { diversidad de } \\
\text { itinerarios / Centrarse } \\
\text { más en la funciones } \\
\text { y en los contenidos } \\
\text { que en las estructuras }\end{array}$ & $\begin{array}{l}\text { "Ingeniería de } \\
\text { itinerarios» tiene } \\
\text { algún efecto, dentro } \\
\text { de los imperativos / } \\
\text { Las políticas } \\
\text { pueden tratar de } \\
\text { las bases racionales } \\
\text { y/o culturales del } \\
\text { comportamiento }\end{array}$ & $\begin{array}{l}\text { Distinguir los } \\
\text { itinerarios reales de } \\
\text { los mapas oficiales / } \\
\text { Adoptar un enfoque } \\
\text { holístico: permitir } \\
\text { otras transiciones / } \\
\text { Proporcionar } \\
\text { indicadores y ayudar } \\
\text { a navegar por los } \\
\text { itinerarios / Aumentar } \\
\text { las opciones y la } \\
\text { diversidad / Medidas } \\
\text { a pequeña escala. }\end{array}$ \\
\hline $\begin{array}{l}\text { Preocupaciones } \\
\text { teóricas subyacentes }\end{array}$ & Análisis societal & $\begin{array}{l}\text { Globalización / } \\
\text { Acción racional } \\
\text { versus explicaciones } \\
\text { "culturalistas» del } \\
\text { comportamiento }\end{array}$ & $\begin{array}{l}\text { Sociedad del riesgo / } \\
\text { Estructura y actuación }\end{array}$ \\
\hline
\end{tabular}


la OCDE, sobre la transición de la educación inicial a la vida laboral, que distinguía entre «tres clases principales de itinerarios a través de la educación secundaria superior, y más allá de ésta, al trabajo o a los estudios terciarios: itinerarios de educación general, itinerarios de formación profesional escolar e itinerarios profesionales de tipo aprendizaje» (OCDE, 2000: 58). Las preguntas políticas generales vinculadas a este concepto de itinerarios incluyen: ¿cuáles son los puntos fuertes y débiles de cada itinerario?, ¿cuál podría ser su magnitud relativa en un sistema de transición que goza de un buen funcionamiento?

El contenido, el papel y la organización de cada itinerario, así como la proporción de gente joven que lo emprende son aspectos que varían mucho de un país a otro. Existe menos variación en otras características de los itinerarios, como su estatus relativo, tal como se indica en el contexto social o educativo de la gente joven que los sigue. En todos los países, los itinerarios académicos gozan de un estatus superior que los itinerarios profesionales en el nivel secundario superior y, en la mayor parte de países, los itinerarios profesionales escolares gozan de un estatus superior que los itinerarios basados en la formación ocupacional o similares. Las mujeres tienden a estar poco representadas en la formación ocupacional, sobrerrepresentadas en los itinerarios profesionales escolares y ligeramente sobrerrepresentadas en los itinerarios académicos, aunque las diferencias de género varían más que las diferencias de clase de un país a otro (OCDE, 2000; Smyth, 2000; Smyth et al., 2001).

La tendencia dominante a largo plazo es el «empuje académico» ${ }^{5}$ - un incremento de la proporción de jóvenes que emprenden itinerarios académicos y una disminución en la proporción de jóvenes que emprenden los itinerarios profesionales y, en particular, los no reglados. Sin embargo, los modelos de participación han fluctuado en el tiempo y han variado de un país a otro. Generalmente, la deriva académica ha sido más débil en aquellos países con mercados laborales más fuertemente estructurados por límites ocupacionales (occupationalized) o con sectores universitarios reducidos (Müller y Wolbers, 2000). El Pathways Study ('Estudio de Itinerarios') de la OCDE (1998) de diez países reveló que, durante la década de 1980, en general, la participación en la educación académica aumentó más rápidamente que la participación en la formación profesional. Exclusivamente en Holanda, la formación profesional incrementó su cuota de participación de forma significativa. Entre los subsectores de la formación profesional, el no reglado disminuyó en todos los países, mientras que el mayor crecimiento se dio en los programas que permitían acceder a la educación superior. La formación profesional tendió a ser empujada hacia arriba desde el nivel secundario superior hasta el sector terciario - en donde a menudo gozó de un estatus muy superior. A similares conclusiones llegaron Green et al. (1999), que identificaron un proceso de empuje académico en toda la Unión Europea entre los años 1984 y 1994. Sostenían que, a menudo, el empuje académico se escondía tras la manera en

5. N. de la t.: en la versión original, academic drift. 
que se clasificaban las vías, y que esto no sólo se reflejaba en las proporciones crecientes de la cohorte de edad que obtenía títulos académicos o generales, sino también en los cambios que tenían lugar en el itinerario profesional que, a menudo, se volvió más académico en sus objetivos y en su naturaleza. Esto podría explicar claras excepciones en esta tendencia; por ejemplo: en Inglaterra, el itinerario profesional escolar se amplió en la década de 1990, inversamente a la tendencia general, pero algunos críticos sostienen que en este proceso perdió su integridad «profesional» (Smithers, 1993).

¿Pero importa el empuje académico? ¿Deberían los encargados de formular las políticas intentar influir en la magnitud relativa y el carácter de los diversos itinerarios postobligatorios? A menudo, los encargados de formular políticas intentan responder a estas preguntas en relación con el mercado laboral: ¿qué itinerarios proporcionan los mejores resultados ocupacionales? Numerosos investigadores han examinado esta cuestión (por ejemplo: Payne, 1995; Van der Velden y Lodder, 1995; Ryan, 1998, 2001; Müller y Shavit, 1998; Brauns et al., 1999; Sofer, 1999; Dearden et al., 2001; Smyth et al., 2001). Seguidamente intentaré resumir sus conclusiones:

- En todos los países, los itinerarios profesionales, y especialmente los itinerarios no reglados, tienen más posibilidades que los itinerarios generales de conducir directamente al mercado laboral.

- Para las personas que dan sus primeros pasos en el mercado laboral, ni los itinerarios generales ni los profesionales están asociados de forma consistente a obtener mejores resultados en el mercado laboral. Diferentes estudios producen diferentes conclusiones.

- El efecto de seguir un itinerario profesional más que uno académico presenta mayores posibilidades de resultar siendo positivo cuando el criterio es el empleo (versus desempleo), que cuando el criterio son los ingresos o (especialmente) el nivel de cualificación.

- Los resultados del mercado laboral varían de un programa profesional a otro. Los resultados más positivos están asociados a los programas ocupacionalmente específicos, con programas en algunas áreas especializadas como los negocios, con itinerarios que no están estigmatizados para los menos capaces o menos motivados, con una formación que está estrechamente relacionada con el mercado laboral y con la gente que obtiene empleos en el área de su formación.

- En palabras de Shavit y Müller (2001), la formación profesional puede, a la vez, «desviar» a los jóvenes de los empleos de alto nivel o de la formación que conduce a ellos, y proporcionar una «red de seguridad» que los protege del desempleo o del trabajo no cualificado.

- Comparado con los varones, las mujeres obtienen mejores resultados en el mercado laboral de los itinerarios profesionales reglados y resultados más negativos de los itinerarios de formación no reglada.

- Los itinerarios profesionales reglados y no reglados proporcionan de media resultados similares. Los jóvenes que han hecho un curso de formación 
ocupacional o similar tienden a presentar unos índices superiores de primer empleo, pero a menudo encuentran trabajos de menor nivel, y sus oportunidades de progreso y movilidad profesional pueden ser más reducidas.

- Todas estas conclusiones varían de un país a otro, así como de un tipo de programa a otro. La formación profesional tiende a presentar resultados más positivos en el mercado de trabajo en aquellos países con mercados laborales más fuertemente estructurados en base a ocupaciones reglamentadas.

- Las conclusiones de la investigación también varían dependiendo de los conjuntos de datos analizados y de las hipótesis utilizadas en el análisis; por ejemplo: los criterios para decidir qué programas académicos o profesionales están en el mismo «nivel», y que, por lo tanto, son comparables, si el análisis tiene en cuenta la educación postescolar y la formación continua después de abandonar cada uno de los itinerarios, y de qué forma permite el desarrollo de las diferentes características de los estudiantes que emprenden cada itinerario.

Estos resultados ambiguos y la amplia variación transnacional sugieren que contrastar planes nacionales puede ser igualmente factible. Sin embargo, aun cuando su veredicto fuese menos ambiguo, el mercado laboral no sería el tribunal de apelaciones de última instancia sobre los beneficios de los distintos itinerarios. Sus criterios pueden chocar con prioridades tales como la justicia social o la inclusión. Y especialmente en economías con bajas cualificaciones como la del Reino Unido, los analistas políticos han sostenido que las demandas del mercado laboral y las estrategias empresariales que aquéllas reflejan deberían ser ellas mismas objeto de intervención política. En vez de utilizar criterios del mercado laboral para evaluar la efectividad de los itinerarios educativos, las políticas deberían reformar el mercado laboral a la vez que la educación para conseguir la sinergia requerida para una economía emergente con cualificaciones altas (Ashton y Green, 1996; Keep y Mayhew, 2001; Performance and Innovation Unit, 2001). La mayor parte del debate político transnacional sobre los itinerarios se basa en criterios más amplios. Los argumentos para resistir el empuje académico y mantener un itinerario profesional sustancial en el nivel secundario superior incluyen los aspectos siguientes: un itinerario profesional desarrolla cualificaciones para la competitividad; es mejor desde el punto de vista pedagógico, incluso como una forma de adquirir conocimientos «generales» o competencias, porque vincula el aprendizaje teórico al aplicado; motiva a los jóvenes a aprender, especialmente a aquellos que están descontentos con la educación académica, $y$, finalmente, es socialmente más inclusivo. Estos argumentos se aplican tanto a los itinerarios de formación profesional reglada como a los no reglados. Los argumentos específicamente a favor de la formación no reglada incluyen que mantiene la formación próxima a una práctica ocupacional cambiante, que socializa a la gente joven en el puesto de trabajo y que le permite adquirir una identidad ocupacional. 
En la otra cara del debate, los argumentos en contra de llevar a cabo esfuerzos especiales para mantener o desarrollar un itinerario profesional fuerte en el nivel secundario superior incluyen que:

- Deberíamos respetar las preferencias de los jóvenes, incluso en el caso de que éstas tengan como resultado el empuje académico.

- La educación general desarrolla las competencias genéricas, personales e interpersonales de las cuales depende una futura competitividad económica.

- Las actuales necesidades de cualificaciones hacen deseable que se posponga la educación profesional al nivel superior.

- Las cualificaciones profesionales específicas se aprenden mejor cuando los individuos ya trabajan de forma regular, respondiendo a la necesidad y cuando pueden ser reforzadas con la práctica.

- Respecto a la formación no reglada, que la oferta de plazas varía de forma cíclica, es desigual en todas las ocupaciones y está muy sesgada por géneros; que el proceso de aprendizaje puede chocar con las demandas de la producción, y, finalmente, que a menudo la calidad de la formación varía.

El argumento decisivo podría ser que, en la práctica, poco podemos hacer al respecto. Una vez que se ha sobrepasado cierto punto, el empuje académico puede ser irreversible. Los itinerarios profesionales se ven atrapados en un círculo vicioso de estatus y expectativas bajos, y no pueden recuperar fácilmente su prestigio anterior. $\mathrm{Y}$ es que resulta difícil crear itinerarios nuevos o copiar los de otros países. Los intentos para transplantar el sistema dual o algunos aspectos de éste a contextos menos favorables como Estados Unidos han tenido un éxito limitado (Durand-Drouhin et al., 1998; Hamilton y Hamilton, 1999; Stern, 2000).

Ello sugiere que el mejor equilibrio entre itinerarios depende de las circunstancias de cada país y de su situación de partida. Llevado al extremo, este enfoque «societal ${ }^{6}$ puede llevarnos a la conclusión de que cada país es único y de que poco se puede aprender de las comparaciones entre países. Sin embargo, algunos investigadores comparativos han cambiado el lugar del énfasis que antes ponían en intentar identificar la mejor práctica en términos de estructuras institucionales y una mezcla particular de itinerarios, a ponerlo ahora en la exploración de las funciones o las condiciones que cualquier sistema nacional de itinerarios debería satisfacer (Rosenbaum et al., 1990; Van der Velden, 2002). En la misma línea, la OCDE (2000: 13) identificó cinco «ingredientes fundamentales de los sistemas de transición que funcionan correctamente»:

- Una economía sana.

- Itinerarios bien organizados que vinculan la educación inicial al trabajo y la formación posterior.

6. N. de la t.: en la versión original, societal. 
— Oportunidades generalizadas para poder combinar experiencia laboral con formación.

- Densas redes de seguridad para aquellas personas que están en situación de riesgo.

— Instituciones y procesos efectivos.

Típicamente, este enfoque nos lleva a la conclusión práctica de que mantener una diversidad de itinerarios es más importante que mantener una dimensión o un aspecto concretos de un itinerario particular cualquiera. Otra conclusión es que las políticas deberían prestar más atención a lo que sucede en cada itinerario; por ejemplo: un nivel mínimo de competencias cognitivas básicas y de competencias personales y sociales parece ser fundamental para tener éxito en el mundo laboral (Bynner, 1997; Dearden et al., 2001). En algunos países, existe un interés en la idea de una "plataforma mínima de aprendizaje»" que todos los jóvenes deberían alcanzar (McIntosh y Steedman, 1999). Podría ser que, en estas áreas, la evidencia de la investigación fuese más directamente transferible de un país a otro.

\section{Relaciones e interconexiones entre itinerarios}

La segunda utilización del concepto de itinerarios sirve para expresar la relación entre ellos. Las preguntas fundamentales para la investigación y la política son: ¿de qué forma y cuán estrechamente vinculados deberían estar los distintos itinerarios? y ¿de qué forma deberían estar organizados los itinerarios como sistema?

De la misma forma que el «empuje académico» y los esfuerzos para resistirlo proporcionan el contexto de los debates políticos relacionados con la primera utilización del concepto de itinerarios, el proceso de «unificación» y las presiones que conducen a éste proporcionan el contexto para esta utilización del concepto. La «unificación» de la educación postobligatoria y de los sistemas formativos (Raffe, 2002) se refiere a las estrategias que, de forma muy diversa, tienen por objeto:

- Integrar los currículos académicos y profesionales.

- Reducir las diferencias entre vías o itinerarios en la educación postobligatoria, o reunirlas en un «sistema unificado».

- Crear oportunidades perfectamente consistentes para poder tener acceso y progresar en la formación postobligatoria.

Los ejemplos de las tendencias unificadoras incluyen:

- Una mayor coincidencia en los objetivos y en los resultados deseados de los diferentes itinerarios.

7. N. de la t.: en la versión original, minimum learning platform. 
- Convergencia entre itinerarios en sistemas de evaluación y certificación, o en los marcos de cualificaciones que enmarcan los itinerarios.

- Elementos curriculares comunes, como las «cualificaciones clave».

- Instituciones integradas que ofrezcan programas académicos y profesionales.

- Sistemas comunes de gestión, regulación y garantía de calidad.

- Una arquitectura curricular común.

- Itinerarios más flexibles, con cualificaciones duales que conduzcan al empleo y a la educación superior, o con oportunidades para poder cambiar de dirección y transferir créditos.

La unificación se puede interpretar como una respuesta a las presiones económicas y sociales asociadas a la globalización; por ejemplo: demanda de niveles de cualificación superiores o de nuevas competencias que transcienden la distinción entre académica y profesional, presiones para poder disponer de remedios educativos ante el riesgo creciente de exclusión social y económica, o la necesidad de satisfacer las aspiraciones progresivas de los jóvenes y su deseo de mantener las opciones abiertas. No obstante, en muchos países, la principal fuerza impulsora de la unificación se halla en los nuevos imperativos de organización creados por la expansión educativa. A medida que la educación postobligatoria y los sistemas de formación se amplían e incrementan su complejidad funcional, y que sus diversos sectores e itinerarios se vuelven más interdependientes, los primeros requieren una mayor coordinación y coherencia; por ejemplo: los itinerarios independientes que anteriormente conducían directamente al mercado laboral se vuelven callejones sin salida y requieren estar conectados al resto del sistema. Estos imperativos organizativos crean presiones para la «unificación», pero son experimentados de formas distintas según las regulaciones institucionales del sistema. Por lo tanto, la unificación toma diferentes formas en distintos países. Las estrategias nacionales para la unificación varían según los itinerarios que cubren, hasta qué punto los acercan y las características del sistema a las que dan prioridad: instituciones, currículo, certificación, etc. Existe otra diferencia entre sistemas unificados que ponen énfasis en los programas uniformes o comunes, por ejemplo, como base para la ciudadanía, y aquellos que ponen énfasis en la flexibilidad y la diversidad (Lasonen y Young, 1998; Raffe et al., 1998; Spours et al.; 2000; Lasonen y Manning, 2001).

En este debate, el aspecto más importante de la unificación es el desarrollo de sistemas de itinerarios más coherentes, flexibles e interconectados. Esto a menudo se consigue a través de la ingeniería de itinerarios (pathways engineering). (En este punto, la metáfora cambia sutilmente su significado. Lo que hasta ahora hemos descrito como «itinerarios», como los tres amplios itinerarios de la OCDE, se denominan «vías» o «programas»; los «itinerarios» y el «sistema de itinerarios» describen las vías más detalladas a través de y entre ellos.) La ingeniería de itinerarios es un intento deliberado de remodelar los sistemas de itinerarios para garantizar oportunidades adecuadas y favorecer los niveles y los modelos de participación deseados. Típicamente, tiene por objeto atender más 
eficazmente a toda clase de jóvenes, aumentar la participación y la progresión entre aquellos que corren un mayor riesgo de abandonar los estudios y hacer que los itinerarios sean más flexibles respondiendo a las preferencias cambiantes de los jóvenes o las nuevas demandas del mercado laboral. A menudo, tiene por objeto favorecer la participación en los programas profesionales. Los ingenieros de itinerarios pueden, por ejemplo, construir "puentes» desde los programas profesionales hasta la educación superior, para estimular la participación en aquellos programas; pueden ampliar los itinerarios o aplazar los momentos de tomar las decisiones para atraer a la gente que desea mantener abiertas sus opciones; pueden alargar los itinerarios para desbloquear "los callejones sin salida», o, finalmente, pueden introducir medidas tales como la modularización, la transferencia de créditos y los marcos de cualificaciones, al objeto de que la red de itinerarios sea más flexible y más fluida (OCDE, 1998).

Si los ingenieros de itinerarios desean influir en las decisiones de los jóvenes, necesitan comprender de qué forma toman estas decisiones. Un tema clave es hasta qué punto los jóvenes toman «racionalmente» sus decisiones —un tema que aparece en el debate más amplio entre las explicaciones no racionales y de acción racional de las ciencias sociales. Por otra parte, numerosos estudios han demostrado que la gente joven no toma sus decisiones de forma procedimentalmente racional, que las familias, así como los grupos de su edad y las subculturas, influyen en ello, que los comportamientos que aparecen en la toma de decisiones varían de una categoría de jóvenes a otra, y que factores «no racionales», como el desarrollo de las identidades personales y ocupacionales, tienen un papel importante en este proceso. Además, existe una evidencia considerable - a menudo de los mismos estudios- de que las elecciones de itinerarios de los jóvenes son instrumentales, que tienen por objeto el éxito en el mercado laboral, entre otros, y que responden a cambios en los costes y beneficios relativos de escoger diferentes itinerarios educativos. Los resultados totales de las decisiones de los jóvenes reflejan un cálculo racional subyacente, aun cuando los procesos individuales que llevan a tomarlas no lo son: sus decisiones son «pragmáticamente» racionales, cuando no procedimentalmente racionales, e incluso cuando intervienen las subculturas y la presión de sus semejantes, éstas reflejan a su vez una especie de pruebas de la realidad en el tiempo (Gambetta, 1987; Heinz, 1994; Hodkinson et al., 1996; Ball et al., 1999; Furlong, 2002).

La tensión entre las explicaciones no racionales o culturalistas y de acción racional se refleja en los debates políticos sobre la (falta de) paridad de consideración por la formación profesional y la educación académica, uno de los principales problemas tratados por los ingenieros de itinerarios. Normalmente, las explicaciones no racionales o culturalistas atribuyen el poco prestigio de que gozan los itinerarios profesionales a la valoración social de los diversos tipos de conocimientos, y sostienen que la formación profesional goza de un estatus más elevado en unos países que en otros debido a las diversas culturas nacionales. Las explicaciones de la acción racional sostienen que la formación profesional en el nivel secundario superior tiene un estatus bajo porque los rendimientos 
instrumentales son más bajos; tiene menos posibilidades de conducir a la educación superior o a empleos de alto nivel y con sueldos elevados. El enfoque de la acción racional se basa en la evidencia de que el estatus relativo del itinerario profesional es bajo en todos los países, a pesar de las diferentes culturas nacionales; su estatus absoluto puede variar, pero en gran parte respecto a su dimensión — cuanto más amplio es el itinerario profesional, más elevado resulta el nivel educativo de los estudiantes. El estatus relativo de la formación profesional comparado con la educación académica varía mucho de un país a otro, y cuando lo hace se puede explicar por factores «racionales» tales como su importancia cambiante en tanto que vía hacia la educación superior y como una manera de integrarse en el empleo cualificado (OCDE, 1998; Müller y Shavit, 1998).

La ingeniería de itinerarios tiene como recurso principal el elemento racional de la toma de decisiones de los jóvenes. Cambia las oportunidades, los incentivos, los costes y los imperativos asociados a la participación en diferentes programas educativos a fin de poder influir en los modelos de participación y progresión (Raffe, 1998). Pero, ¿¿funciona? Existen claros ejemplos de éxito. Por ejemplo, diversos países han incrementado la participación en los itinerarios profesionales proporcionando puentes a la educación superior o extendiendo los itinerarios profesionales a niveles superiores de estudios (OCDE; 1998, 2000; Lasonen y Manning, 2001). Las oportunidades de racionalización curricular, como en la reforma noruega de 1994, ha proporcionado claros beneficios (McKenzie et al., 1998). La reforma danesa de la formación y la educación profesional del año 2000 se basa en el enfoque de la ingeniería de itinerarios, pero aún es demasiado pronto para valorar sus logros (Nielsen, 2000).

Sin embargo, la ingeniería de itinerarios no es una solución total, y los ingenieros de itinerarios se enfrentan a diversos problemas generales. En primer lugar, sólo pueden controlar partes del sistema. Algunos sectores de la educación, como las universidades, pueden estar más allá de su control directo, y no pueden influir fácilmente sobre la contratación de empleados a partir de diferentes itinerarios, lo cual sería una fuente importante de oportunidades e incentivos. Las reformas en base a módulos de Escocia en la década de 1980 crearon una nueva "lógica intrínseca» de itinerarios flexibles para estimular el acceso y la progresión. No obstante, sólo tuvieron un impacto limitado en los modelos de participación de los jóvenes, que siguieron estando bajo la influencia de una «lógica institucional» más fuerte que surgió de la actividad de las instituciones educativas, el mercado laboral y el contexto social (Raffe et al., 1994); estaban más allá del control de la ingeniería de itinerarios.

En segundo lugar, en una época de cambios rápidos, los destinos de los diversos itinerarios se tornan más ambiguos y el poder de los incentivos mengua. Una respuesta es poner más énfasis en la información y la orientación en las políticas de transición actuales.

En tercer lugar, dado que la educación es un bien posicional, que se ocupa de seleccionar jóvenes para posiciones en el mercado laboral, los ingenieros de itinerarios se enfrentan, pues, al problema de suma cero. Sería posible reestruc- 
turar incentivos para garantizar un «mejor» modelo de participación, pero, en el último análisis, la reserva de incentivos es finita. Ésta es la razón por la cual los encargados en todo el mundo de formular las políticas han encontrado tan difícil idear programas de formación que ofrezcan incentivos creíbles a los jóvenes más desfavorecidos. Éste podría ser un argumento para recurrir a las bases tanto no racionales como racionales del comportamiento; por ejemplo: volver a etiquetar los planes de formación como "aprendizajes», evocando símbolos positivos tales como «bachillerato» o utilizando la formación profesional para desarrollar las identidades personales y ocupacionales.

No obstante - y éste constituye el cuarto problema-, en un sistema de itinerarios flexible, la formación profesional está menos estrechamente vinculada a los destinos ocupacionales y está menos aislada del espíritu académico dominante. Esto hace más difícil que la formación profesional pueda ayudar a algunos jóvenes a desarrollar identidades ocupacionales o a mantener un espíritu vocacional particular. Pero se trata de maneras en que la educación profesional puede favorecer la inclusión social. Uno de los desafíos principales para la política es sostener las características positivas de la unificación y de itinerarios flexibles, a la vez que retener y fomentar una verdadera diversidad. En parte es por ello que los países intentan, aunque parezca increíble, mantener o desarrollar los itinerarios de formación no reglada basada en el aprendizaje directo. Estos programas son más resistentes que otros programas profesionales a la asimilación a una cultura académica dominante; sin embargo, en muchos sistemas, esto también puede provocar que sean más marginales.

\section{Itinerarios y navegación}

La tercera utilización del concepto de itinerarios es el análisis de la relación entre itinerarios como oportunidades estructuradas y la utilización activa de ellos por parte de los jóvenes. Esta relación se expresa mediante las metáforas complementarias de «itinerarios» y «navegaciones». Mientras que el concepto de itinerarios refleja una perspectiva de formulación de políticas, el concepto de navegaciones refleja la perspectiva de individuos que utilizan itinerarios. Por lo tanto, esto halla su eco en el individualismo creciente de las políticas de transición de muchos países. Estas políticas responden a la diversidad cada vez mayor de las necesidades de los jóvenes, a sus elevadas expectativas de elección y a la «individualización» de las transiciones de los jóvenes (Furlong y Cartmel, 1997). También tienen por objeto atribuir un mayor control a los jóvenes en el proceso de transición. Esto se ha convertido en el tema principal de la política en muchos países. Surge a partir de diferentes impulsos: por un lado, el deseo de conferir poder a los jóvenes, de darles control sobre sus vidas y ayudarles a desarrollarse como ciudadanos activos, $y$, por el otro, el deseo de hacer que los individuos, más que los gobiernos o los empleadores, sean responsables de la gestión (e, implícitamente, la financiación) de sus trayectorias educativas y laborales. En palabras del memorándum de la Comisión Europea sobre formación continua, "Vivir y trabajar en la sociedad del conocimiento 
requiere ciudadanos activos que estén automotivados para perseguir su propio desarrollo personal y profesional» (Comisión Europea, 2000: 17). La ciudadanía activa se estimula a través de los procesos educativos, dejando que los jóvenes sean dueños y gestionen sus biografías académicas, así como a través de sus contenidos formales.

Desde la perspectiva política, itinerarios y navegaciones son conceptos complementarios. La metáfora de las navegaciones no desplaza los itinerarios, tal como la periodización de Evans y Furlong podría sugerir; se trata más bien de que los itinerarios deben ser diseñados para responder a las necesidades de los jóvenes que los utilizan. Las preguntas de política e investigación incluyen: primero, ¿de qué forma los itinerarios pueden reflejar las prioridades y las perspectivas de los jóvenes?, y, segundo, ¿de qué forma se pueden organizar los itinerarios para permitir que cada joven mantenga un control activo sobre sus propias transiciones y, naturalmente, construir sus propios itinerarios?

Empezaré con la primera y más fácil de estas preguntas. Si concebimos los itinerarios y las navegaciones como conceptos complementarios, podemos identificar por lo menos tres formas de colaborar a conseguir que los itinerarios reflejen las prioridades y las perspectivas de los jóvenes. La primera es asegurarnos de que los «mapas» oficiales de itinerarios en los que se basan las políticas corresponden a las maneras en que realmente se experimentan y se utilizan los itinerarios. Si esto no es así, las políticas basadas en ellos tendrán fallos. Por ejemplo, muchas políticas de formación para los parados fracasan porque tienen como premisa los itinerarios ideales expresados por la retórica oficial; normalmente, éstos exageran la importancia del capital humano como un medio para alcanzar un empleo estable e infravaloran la importancia de las redes y del capital social. Para crear políticas efectivas, tenemos que comprender los itinerarios «reales» vividos por los jóvenes. Los mapas de itinerarios oficiales pueden ser imprecisos cuando se dan cambios rápidos, como en Europea del Este, en donde los «mapas» heredados del pasado o tomados prestados a Occidente pueden resultar ser guías engañosas para la política (Roberts, 1996, 1998). Los mapas oficiales de itinerarios tienden a dejar de lado las transiciones menos institucionalizadas. En Japón, las políticas de transición han tomado como premisa los itinerarios reconocidos oficialmente desde un sistema educativo estructurado hasta un sistema de empleo para toda la vida; en este sistema, se tiende a pasar por alto a los estudiantes que abandonan la escuela, a los licenciados que encuentran trabajo a través de vías no institucionalizadas y a aquellos que abandonan sus primeros empleos; sobre ellos se recoge poca información (Ihm et al., 2000). Recientemente, un grupo de investigadores europeos observó algo similar: las políticas basadas en normas de transición anticuadas pueden conducir a la exclusión social si ignoran la diversidad de modelos de transición individualizados o "desestandarizados» (European Group for Integrated Social Research, 2001). A veces, los encargados de formular políticas intentan modificar los itinerarios para hacer encajar sus mapas, y no a la inversa. En Holanda, el mapa oficial de los itinerarios muestra que los que son académicos y profesionales ascienden de forma paralela, mientras 
que las transiciones reales presentan un movimiento mucho más oblicuo (De Bruijn y Voncken, 1998). Esta discrepancia ha sido reconocida desde hace tiempo, pero las políticas actuales tienen por objeto reforzar las trayectorias de progresión vertical; en otras palabras, hacer que los itinerarios encajen con el mapa oficial (Nijhof, 2001).

En segundo lugar, los itinerarios deben permitir diferentes clases de transición. A la vez que los jóvenes se van abriendo camino a través de la educación inicial y situándose en el mercado laboral, también pueden estar buscando una mayor independencia de sus padres, estableciendo nuevas relaciones, experimentando nuevos estilos de vida, uniéndose a nuevos hogares, teniendo hijos, enfrentándose a problemas de salud, etc. Y, a medida que los itinerarios se alargan, estas diferentes transiciones se vuelven cada vez más interdependientes. Un cambio en la relación con los propios padres, el nacimiento del primer hijo o el principio de una mala salud son factores que pueden tener un impacto enorme en las transiciones de una persona joven al mundo laboral (Bynner et al., 1997). Los itinerarios tienen que ser diseñados de forma flexible para poder dar cabida a estos acontecimientos, así como a una variedad de «incidentes críticos» menos transcendentes que pueden influir en las transiciones (Gallacher et al., 2000). El resultado de todo ello es que, en muchos países, estamos viendo una convergencia de los principios para diseñar itinerarios para jóvenes y para adultos, típicamente en el marco de una formación continua. La interdependencia de diferentes transiciones también significa que la investigación y la política relacionadas con los itinerarios deberían ser holísticas en su alcance y deberían reconocer la necesidad de disponer de intervenciones por parte de múltiples organismos y la formulación de políticas "con una mayor coherencia» (Eldridge, 2001).

En tercer lugar, los itinerarios requieren paneles de indicadores, y la gente joven debe aprender cómo navegar. La longitud y la complejidad creciente de los itinerarios, así como la velocidad a la que están cambiando, no sólo hace que la información y la orientación sean más importantes, sino que apuntan nuevos modelos de orientación continua, a lo largo de toda la vida, con un centro de atención más holístico y nuevas relaciones entre las fuentes de orientación formales e informales. El memorándum de la Comisión Europea sobre la formación continua reclama «un nuevo enfoque [...] que conciba la orientación como un servicio permanentemente accesible a todos y que supera la distinción entre orientación educativa, profesional y personal, y que trate de llegar a nuevos públicos». Este enfoque se centra en el cliente y explota vías menos formales e informales, a fin de mejorar el acceso de los grupos desfavorecidos que constituyen el objetivo de aquélla (Comisión Europea, 2000: 17).

La segunda pregunta a la que esta utilización del concepto de itinerarios se dirige es: ¿¿de qué forma se pueden diseñar los itinerarios al objeto de realzar el papel activo de los jóvenes en la configuración de sus propias transiciones? Esta pregunta es más difícil de responder sobre la base de la investigación, en parte porque incurre en una pregunta más amplia: ¿̇e qué manera podríamos reconocer si los jóvenes han adquirido más control sobre sus transiciones? Mencionaré brevemente tres formas posibles de «investir de poder» a los jóvenes. 
La primera es incrementar sus opciones: ofrecerles itinerarios más largos, más diversos y más flexibles, con más momentos de decisión. Ésta es la tendencia de las políticas recientes sobre itinerarios. La reforma danesa de 2000 ofrece «itinerarios flexibles para cada estudiante» y tiene por objeto alcanzar un «nuevo equilibrio entre el sistema y el individuo» (Danish Ministry of Education, 2001: 14, 17). En otros países como Australia, los itinerarios flexibles ya sustentan transiciones muy diferenciadas e individualizadas (McKenzie, 2000). Sin embargo, el hecho de que los itinerarios estén cada vez más diferenciados no constituye, en sí mismo, una evidencia de que los jóvenes tengan más control sobre ellos. Y aún menos significa que tengan más control sobre las cosas que más cuentan para ellos, como su nivel ocupacional cuando (y si) consiguen puestos relativamente estables en el mercado laboral. Los investigadores que analizan a los jóvenes utilizan el término «individualización estructurada» para describir una situación en la que las transiciones están más individualizadas, en el sentido de que están diferenciadas, aunque la influencia subyacente de la estructura social, tal como se refleja por ejemplo en las desigualdades de clase, género y etnicidad, sigue siendo fuerte (Roberts et al., 1994). Según esta visión, la individualización es ampliamente subjetiva; refleja una retórica política individualista que desvía la responsabilidad de los fracasos del sistema hacia los individuos que sufren sus consecuencias (Furlong y Cartmel, 1997).

Un segundo mecanismo posible para «investir de poder» a los jóvenes es darles poder en el mercado. Un ejemplo de ello lo encontramos en el breve experimento del Reino Unido llevado a cabo en la década de 1990 con los youth credits (Nota del T.: créditos para jóvenes que tienen un valor que puede ser intercambiado por formación a través de un empresario o un proveedor de formación reconocido) que podían gastar en formación aquellos estudiantes que abandonaban tempranamente la escuela a través de un empleador. Se suponía que los créditos incrementaban su poder en el mercado, pero la política hizo suposiciones nada realistas, tanto sobre el poder de negociar de los jóvenes con los empleadores como sobre su fuerza global en el mercado. La medida fue concebida en una época en que la mano de obra juvenil era escasa y los jóvenes tenían potencialmente poder en el mercado laboral, pero cuando finalmente aquélla se implementó, el paro juvenil estaba aumentando de nuevo. Una política basada en el mercado puede no ser la mejor forma de dar poder a un grupo cuyo problema básico es su debilidad en el mercado.

La tercera forma posible de otorgar poder a los jóvenes es simplemente diseñando itinerarios de tal forma que reflejen las propias perspectivas y prioridades de los jóvenes, en las distintas maneras ya descritas, y proporcionando los recursos - materiales, culturales y sociales - que la gente joven necesita para navegar por ellos. Este enfoque es menos ambicioso en el sentido de que se puede reflejar en un gran número de pequeñas cosas más que en un único movimiento espectacular de la varita mágica del mercado, pero puede ser más efectivo. En realidad, equivale a tratar a los individuos y a sus navegaciones como un aspecto fundamental del concepto de itinerarios. 


\section{Conclusión: puntos fuertes y débiles del concepto de itinerarios}

En este artículo, he intentado demostrar que la metáfora de los itinerarios puede constituir un concepto de organización útil en los debates actuales sobre las transiciones de la educación al trabajo, así como un puente entre la investigación y la política. He diferenciado tres utilizaciones principales del concepto de itinerarios que no sólo corresponden a áreas de investigación empírica en las transiciones de los jóvenes, sino también a algunas de las preguntas teóricas subyacentes de las ciencias sociales. Las tres utilizaciones del concepto de itinerarios no son ni una secuencia cronológica ni una secuencia lógica. No he intentado sugerir que alguno de estos conceptos supere a los demás o que deba substituirlos.

He sugerido que aquellos que utilizan la metáfora de los itinerarios deberían ser más precisos respecto a la manera en que la utilizan, aunque se trate de una metáfora; no es ni una teoría ni una metodología para el análisis político. Tampoco describe una corriente de investigación particular. Una parte muy pequeña de la investigación analizada en este artículo lleva una etiqueta explícita de «itinerarios» y recorre una diversidad de disciplinas, tradiciones y paradigmas. Esto dificulta el hecho de poder juzgar si las críticas del concepto de itinerarios, y especialmente de la «investigación de los itinerarios» son justas. Al principio de este artículo, me refería a las críticas de la linealidad, el economicismo y el individualismo. De éstas, lo más fácil es defender el concepto de itinerarios ante la crítica de la linealidad. En la medida en que la investigación de los itinerarios ha asumido que las transiciones son lineales, esto refleja ampliamente las limitaciones de las fuentes de datos disponibles. La insuficiencia de la evidencia empírica sobre las transiciones de los jóvenes está extensamente reconocida (Roberts, 1997; Raffe, 2001; Sweet, 2001). Estos datos que están disponibles tienden a tener una capacidad limitada para medir el cambio social a través de un registro longitudinal. Una consecuencia es que las transiciones al mundo laboral se representan en términos «lineales»-como una única transición, de la formación a tiempo completo a la participación a tiempo completo en el mercado laboral, con ningún punto de coincidencia y ningún estatus intermedio- - ya que esto exige menos datos longitudinales, y porque simplifica las comparaciones transnacionales. Por ejemplo, los Key Data ('datos clave') de la Comisión Europea sobre las transiciones de la escuela al trabajo, basados sobre todo en la European Labour Force Survey (ELFS) (Encuesta Europea de Población Activa), muestra un modelo lineal de una única transición (CEDEFOP, 2001); lo mismo sucede con el módulo sobre la transición de la escuela al trabajo añadido al 2000 ELFS, para el cual los servicios estadísticos nacionales recibieron la orden de «corregir» la fecha de inicio del primer empleo, de tal forma que no antecediese a la fecha de abandono de la educación inicial.

Dentro de las limitaciones de las fuentes de datos disponibles, diversos estudios han examinado los modelos de transición no lineales (McKenzie, 2000; Wolbers, 2001; Furlong et al., 2001). No existe nada en el propio concepto de itinerarios que predisponga a un modelo lineal de transiciones de los jóvenes. 
Tal como Evans y Furlong (1997) apuntan, el concepto se fue volviendo más popular en la década de 1980, porque describía los modelos más largos y más complejos de transiciones que estaban surgiendo por aquel entonces. En países tales como Australia y Dinamarca, el concepto de itinerarios se ha asociado progresivamente a políticas para incrementar la flexibilidad y, por lo tanto, para adaptar las transiciones no lineales. A menudo, está relacionado con la retórica política de la formación continua, la cual pretende fundamentalmente ajustar la oferta educativa a los modelos de transición no lineales.

La segunda crítica del concepto de itinerarios, que es economicista, es válida en el sentido de que gran parte del debate político sobre los itinerarios (y la mayor parte de la investigación que examinamos en este artículo) se ocupa de las transiciones a y en el mercado laboral. Como sucedía con la crítica de la linealidad, esto refleja en parte las limitaciones de los datos: muchos de los conjuntos de datos (y, en particular, los conjuntos de datos transnacionales) contienen poca información sobre otros tipos de transiciones. No obstante, el economicismo de los debates políticos sobre los itinerarios también refleja las responsabilidades políticas de aquellos que utilizan el concepto. El mismo concepto de itinerarios no excluye un enfoque holístico que reconoce la interdependencia de las transiciones de la educación al trabajo de las transiciones de familia, vivienda y estilos de vida. El informe reciente del Australian Taskforce on Youth Pathways constituye un ejemplo de este enfoque (Eldridge, 2001).

La tercera crítica, que el concepto de itinerarios pasa por alto la estructura social, es más sustanciosa. Por contraste con el concepto de «trayectorias», la metáfora de los itinerarios centra la atención en factores estructurales que están bajo el control directo de los encargados de formular las políticas: el diseño de los itinerarios. Las mismísimas connotaciones de la elección individual y la libertad para navegar, que hacen que la metáfora de los itinerarios sea atractiva en primer lugar para los encargados de formular las políticas, pueden distraer su atención de las maneras en que los trayectos reales de los jóvenes están determinados por factores tales como clase, género y etnicidad. La retórica de los itinerarios puede llevar a los responsables de formular las políticas a percibir la desventaja como un problema individual que se tiene que tratar mediante itinerarios flexibles que responden mejor a las necesidades individuales. Pero la flexibilidad puede incrementar la desventaja de aquellos jóvenes que, por ejemplo, no tienen los recursos necesarios para llevar a cabo los trayectos más largos que los itinerarios flexibles permiten, o para imponerse en los planes más regidos por el mercado que algunas políticas para la flexibilidad pueden estar sustentando.

Los responsables de formular las políticas y los analistas que utilizan el concepto de itinerarios no necesariamente ignoran la estructura social; el Thematic Review de la OCDE prestaba una atención considerable a las desigualdades de género. No obstante, aquellos que utilizan cualquier clase de metáfora deberían ser conscientes de los significados no deliberados que ésta puede transmitir. El concepto de itinerarios resulta útil como concepto general estructurador y como puente entre la investigación y la política, pero la metáfora no debería acabar con el análisis. 


\section{Agradecimientos}

Se presentó una primera versión de este artículo como un texto que estableció la tónica en la conferencia de investigación del Australian Council for Educational Research, celebrada en Melbourne en octubre de 2001. Cathy Howieson hizo comentarios muy útiles sobre una primera versión.

Traducción al español: Beatriz Krayenbühl Gusi.

\section{Referencias bibliográficas}

Ashton, D. y Green, F. (1996). Education, Training and the Global Economy. Cheltenham: Edward Elgar.

Ball, S.; Macrae, S. y MaGuire, M. (1999). «Young lives, diverse choices and imagined futures in an education and training market». International Journal of Inclusive Education, 3 (3), 195-224.

Brauns, H.; GANGL, M. y SCHERER, S. (1999). «Education and unemployment: patterns of labour market entry in France, the United Kingdom and West Germany». En: RAFFe, D.; VAN DER Velden, R. y Werquin, P. (eds.). Education, the Labour Market and Transitions in Youth: Cross-National Perspectives. Edimburgo: CES, Universidad de Edimburgo.

Bruijn, E. de y Voncken, E. (1998). «The Netherlands». En: OECD (ed.). Pathways and Participation in Vocational and Technical Education and Training. París: OCDE.

BYNNER, J. (1997). «Basic skills, employability and accelerating social exclusion». En: Economic and Social Research Institute and Combat Poverty Agency (eds.). Transitions in Youth: Combating Exclusion. Dublín: Proceedings of the Fourth European Workshop.

BYNNER, J.; FerRI, E. y SHEPHERD, P. (1997). Twenty-Something in the 1970s. Aldershot: Ashgate.

CEDEFOP (2001). The Transition from Education to Working Life: Key Data on Vocational Training in the European Union. Luxemburgo: Office for Official Publications of the European Communities.

Cohen, P. y AinleY, P. (2000). «In the country of the blind?: Youth studies and cultural studies in Britain». Journal of Youth Studies, 3 (1), 79-95.

Danish Ministry of Education (2001). New Structures in the Danish Vocational Education and Training. Copenhague: Ministerio Danés de Educación.

Dearden, L.; Mcintosh, S.; Myck, M. y Vignoles, A. (2001). "The returns to academic, vocational and basic skills in Britain». Skills Task Force Research Paper. Londres: CEP, London School of Economics.

DEARING, R. (1996). Review of Qualifications for 16-19 Year Olds. Hayes: SCAA Publications.

Durand-Drouhin, M.; MCKenZie, P. y SweEt, R. (1998). «Opening pathways from education to work». OECD Observer, 214.

DWYER, P. y WYN, K. (1998). «Post-compulsory education policy in Australia and its impact on participant pathways and outcomes in the 1990s». Journal of Education Policy, 13 (3): 285-300.

ELDRIDGE, D. (Chair) (2001). Footprints to the Future: Report from the Prime Minister's Youth Pathways Action Plan Taskforce 2001. Canberra: AusInfo. 
ERTL, H. (2002). "The concept of modularisation in vocational education and training: the debate in Germany and its implications». Oxford Review of Education, 28 (1), 53-73.

European Commission (2000). A Memorandum on Lifelong Learning, SEC(2000)1832. Bruselas: Comisión Europea.

European Group for InTEgrated Social ReSEARCH (2001). «Misleading trajectories: transition dilemmas of young adults in Europe». Journal of Youth Studies, 4 (1), 101-118.

Evans, K. y Furlong, A. (1997). "Metaphors of youth transitions: niches, pathways, trajectories or navigations». En: Bynner, J.; Evans, K. y Furlong, A. (eds.). Youth, Citizenship and Social Change in a European Context. Aldershot: Ashgate.

Furlong, A. (2002). Accounting for Agency in Modern Youth Transitions. Glasgow: Universidad de Glasgow, Departamento de Sociología.

Furlong, A. y Cartmel, F. (1997). Young people and Social Change: Individualisation and Late Modernity. Buckingham: Open University Press.

Furlong, A.; Cartmel, F.; Biggart, A.; Sweeting, H. y West, P. (2001). Working Papers. Project on Reconceptualising Youth Transitions. Glasgow: Universidad de Glasgow.

Gallacher, J.; Crossan, B.; Leahy, J.; Merrill, B. y Field, J. (2000). Education for All? Glasgow: Glasgow Caledonian and Stirling Universities. Centre for Research in Lifelong Learning.

Gambetta, D. (1987). Were they Pushed or Did they Jump?: Individual Decision Mechanisms in Education. Cambridge: Cambridge University Press.

Green, A.; Wolf, A. y Leney, T. (1999). Convergence and Divergence in European Education and Training Systems. Bedford Way Papers. Londres: University of London Institute of Education.

Hamilton, S. y Hamilton, M. (1999). «Creating new pathways to adulthood by adapting German apprenticeship in the United States». En: HeINZ, W. (ed.). From Education and Work: Cross-National Perspectives. Cambridge: Cambridge University Press.

HeInZ, W. (1994). "Transition behaviour and career outcomes in England and Germany». En: CEDEFOP (ed.). The Determinants of Transitions in Youth. Berlín: CEDEFOP.

Hodkinson, P.; Sparkes, A. y Hodkinson, H. (1996). Triumphs and Tears: Young People, Markets and the Transition from School to Work. Londres: David Fulton.

Ihm, C.-S.; Jappinen, A.; Mckenzie, P. y Raffe, D. (2000). Thematic Review of the Transition from Initial Education to Working Life. Country Note: Japan. París: OCDE.

KeEP, E. y MAYHEW, K. (2001). "Globalisation, models of competitive advantage and skills». SKOPE Research Paper No 22. Coventry: Universidades de Oxford y Warwick.

Kirby, P. (Chair) (2000). Ministerial Review of Post Compulsory Education and Training Pathways in Victoria. Melbourne: Estado de Victoria.

LASONEN, J. y MANNING, S. (2001). «How to improve the standing of vocational compared to general education: A collaborative investigation of strategies and qualifications across Europe». En: DESCY, P. y TESSARING, M. (eds.). Training in Europe: Second Report on Vocational Training Research in Europe 2000: Background Report. Luxemburgo: Office for Official Publications of the European Communities. 
Lasonen, J. y Young, M. (1998). Strategies for Achieving Parity of Esteem in European Upper Secondary Education. Jyväskylä: Universidad de Jyväskylä, Institute for Educational Research.

Mcintosh, S. y Steedman, H. (1999). Low Skills: A Problem for Europe. Londres: Centre for Economic Performance, London School of Economics.

Mckenzie, P. (2000). "Pathways for youth in Australia». Artículo para la Conference on Vocational Training and Lifelong Learning en Australia y Alemania, Potsdam.

Mckenzie, P.; Schmid, E.; STERn, D. y Sweet, R. (1998). Thematic Review of the Transition from Initial Education to Working Life. Country Note: Norway. París: OCDE.

MÜLlER, W. y SHAVIT, Y. (1998). «The institutional embeddedness of the stratification process». En: SHAVIT, Y. y MÜller, W. (eds.). From School to Work. Oxford: Clarendon Press.

MÜlLER, W. y WOLBERS, M. (2000). «Educational attainment of young people in the European Union: cross-country variation of trends over time». CATEWE Working Paper. MZES, Universidad de Mannheim.

NiELSEN, S. (2000). «Analysing strategies for improving vocational education: Towards a framework for European comparisons in Denmark: VET Reform 2000». En: STENSTROM, M.-J. y LASONEN, J. (eds.). Strategies for Reforming Initial Vocational Education and Training in Europe. Jyväskylä: Universidad de Jyväskylä, Institute for Educational Research.

NiJHOF, W. (2001). "The VET system between private demands and public interests: The Dutch challenge». En: Nieuwenhuis, L. y NijHOF, W. (eds.). The Dynamics of VET and HRD Systems. Enschede: Twente University Press.

OECD (1998). Pathways and Participation in Vocational and Technical Education and Training. París: OCDE.

OECD (2000). From Initial Education to Working Life: Making Transitions Work. París: OCDE.

PAYNe, J. (1995). Options at 16 and Outcomes at 24: A comparison of Academic and Vocational Education and Training Routes. Sheffield: Department for Education and Employment.

Performance and Innovation Unit (2001). In Demand: Adult Skills for the 21st Century. [www.cabinetoffice.gov.uk/innovation/2001/workforce/report]

RAFFE, D. (1998). "Where are pathways going?: Conceptual and methodological lessons from the pathways study». En: OECD (ed.). Pathways and Participation in Vocational and Technical Education and Training. París: OCDE.

- (2001). «Strategies for collecting cross-national data on education-to-work transitions». En: RAFFE, D. (ed.). Comparative Data on Education-to-Work Transitions: Report of an International Workshop. [http://www.mzes.uni-mannheim.de/projekte/ catewe]

- (2002). «Bringing academic education and vocational training closer together». Artículo para el Symposium on Futures of Education: Arbeit, Bildung und Beruf. Universidad de Zurich.

Raffe, D.; Croxford, L. y Howieson, C. (1994). "The third face of modules: gendered patterns of participation and progress in Scottish vocational education». British Journal of Education and Work, 7 (3), 87-104.

Raffe, D.; Howieson, C.; Spours, K. y Young, M. (1998). "The unification of post-compulsory education: towards a conceptual framework». British Journal of Educational Studies, 46 (2), 169-187. 
Roberts, K. (1996). «Individualisation and risk in east and west Europe». En: Helve, H. y BYNnER, J. (eds.). Youth and Life Management: Research Perspectives. Helsinki: Helsinki University Press.

- (1997). "Structure and agency: the new youth research agenda». En: BYNNER, J.; Evans, K. y Furlong, A. (eds.). Youth, Citizenship and Social Change in a European Context. Aldershot: Ashgate.

ROBERTS, K. (1998). «School-to-work transitions in former communist countries». Journal of Education and Work, 11 (3), 221-238.

Roberts, K.; Clark, S. y Wallace, C. (1994). «Flexibility and individualisation: a comparison of transitions into employment in England and Germany». Sociology, 28, 31-54.

Rosenbaum, J.; Kariya, T.; Settersen, R. y Maier, T. (1990). «Market and network theories of the transition from high school to work: their application to industrialised societies". Annual Review of Sociology, 16, 263-299.

RYAN, P. (1998). "Is apprenticeship better?: A review of the economic evidence». Journal of Vocational Education and Training, 50 (2), 289-325.

- (2001). "The school-to-work transition: a cross-national perspective». Journal of Economic Literature, 39, 34-92.

SHAVIT, Y. y MÜLLER, W. (2001). "Vocational secondary education: where diversion and where safety net?». European Societies, 2 (1), 29-50.

Smithers, A. (1993). All Our Futures. Londres: Channel 4 Television.

SMYTH, E. (2000). «Gender differentiation in education and early labour market outcomes over time: a comparative analysis». CATEWE Working Paper. Dublín: ESRI.

Smyth, E.; Gangl, M.; Raffe, D.; Hannan, D. y Mccoy, S. (2001). A Comparative Analysis of Transitions from Education to Work in Europe (CATEWE): Final Report. Dublín: ESRI. [http://www.mzes.unimannheim.de/projekte/catewe/]

SofER, C. (1999). «Schooling, training and transitions». Artículo para la European Research Network on Transitions in Youth. Oslo.

Spours, K.; Young, M.; Howieson, C. y Raffe, D. (2000). «Unifying academic and vocational education in England, Wales and Scotland». En: Coffield, F. (ed.). Differing Visions of a Learning Society: Research Findings Volume 1. Bristol: Policy Press.

STERN, D. (2000). "Improving pathways in the United States from high school to college and career». En: OECD (ed.). Preparing Youth for the 21st Century. París: OCDE.

SWEET, R. (2001). "A comprehensive framework for indicators of the transition from initial education to working life: perspectives from the OECD Thematic Review». En: Raffe, D. (ed.). Comparative Data on Education-to-Work Transitions: Report of an International Workshop. [http://www.mzes.unimannheim.de/ projekte/catewe/]

VAN DER VELDEN, R. (2002). Educational Systems and the School-to-Work Transition: A Conceptual Framework. Maastricht: ROA, Universidad de Maastricht.

VAN DER VELDEN, R. y LODDER, B. (1995). "Alternative routes from education to the labour market: labour market effects of full-time versus dualised education». Educational Research and Evaluation, 1 (2), 109-128.

Wolbers, M. (2001). «Learning and working: double statuses in youth transitions within the European Union». CATEWE Working Paper. Maastricht: ROA, Universidad de Maastricht. 
